\title{
Dynamic Response of Buried Heterostructure and Stripe Geometry $\lambda / 4$ DFB Semiconductor Lasers
}

\author{
Y.L. Ling, S.F. Yu and E.H. Li \\ Department of Electrical and Electronic Engineering, The University of Hong Kong, \\ Pokfulam Road, Hong Kong
}

\begin{abstract}
A comparison between different lateral confinement structures in DFB laser is analyzed with identical material parameters and structure in transverse and longitudinal directions. Results show that stripe geometry DFB lasers offer better dynamic response than buried heterostructure DFB lasers.
\end{abstract}

It was indicated that carrier diffusion has significant influence on the damping rate, especially for stripe geometry lasers [1] which suffer high damping of relaxation oscillations. However, this may has a positive effect on the dynamic response of the devices. According to rate equation analysis of Fabry Perot laser, the relaxation oscillation frequency, $\omega_{\mathrm{r}}$, and damping rate, $\gamma$, may be related as $\gamma=\mathrm{K} \omega_{\mathrm{r}}{ }^{2}+1 / \tau_{\mathrm{c}}$, where $\tau_{\mathrm{c}}$ is the carrier lifetime and $\mathrm{K}$ is the damping factor [2]. This implies that the relaxation oscillation frequency of stripe geometry devices may be larger, due to a higher damping, than the other laser structures. Furthermore, it is expected that the dynamic response of DFB lasers are different to Fabry Perot lasers because of the longitudinal spatial hole burning effect. In this paper, we compare the small signal dynamic response of buried heterostructure and stripe geometry in $\lambda / 4$ DFB laser structure including the effects of carrier spatial hole burning and time dependent lateral field profile.

Figure 1 shows the end view of buried heterostructure and stripe geometry $1.55 \mu \mathrm{m}$ InGaAsP DFB lasers with second-order grating. We considered the devices with $\lambda / 4$ phase shifted and both facets anti-reflection coated. The cavity length is $400 \mu \mathrm{m}$ long and the width of the active region is $5 \mu \mathrm{m}$ wide. The differential gain in active and absorption regions and the corresponding transparent carrier densities are given as $3.0 \times 10^{-16} \mathrm{~cm}^{2}$ and $1.2 \times 10^{-15} \mathrm{~cm}^{2}, 1.5 \times 10^{18} \mathrm{~cm}^{-3}$ and $1.7 \times 10^{18} \mathrm{~cm}^{-3}$, respectively. The carrier lifetime is equal to $3 \mathrm{~ns}$ and the effective diffusion constant is set to $8.5 \mathrm{~s}^{-1} \mathrm{~cm}^{2}$. The gain compression factor is $2 \times 10^{-17} \mathrm{~cm}^{3}$. For second order grating, the radiation loss coefficient is equal to $7 \mathrm{~cm}^{-1}$. The total absorption and scattering losses in the waveguide is $20 \mathrm{~cm}^{-1}$ and the linewidth enhancement factor is 4.86 .

Figure 2 shows the small signal modulation response of both buried heterostructure and stripe geometry in $\lambda / 4 \mathrm{DFB}$ lasers with $\kappa=20$ and $50 \mathrm{~cm}^{-1}$, respectively. The lasers are initially biased to give a steady state output level (approximately $3.4 \mathrm{~mW}$ ), the AM and FM response are estimated from the impulse response of the lasers $[3,4]$. It is shown in figure 2(a) that the stripe geometry lasers have larger relaxation oscillation frequency and wider AM bandwidth than buried 
heterostructure devices especially for large $\kappa$. The AM bandwidth is enhanced by more than $3 \mathrm{GHz}$ for devices with $\kappa=50 \mathrm{~cm}^{-1}$. The increment of $\mathrm{AM}$ bandwidth has a value close to that using antiphase complex coupled structure [5]. The FM response are shown in figure 2(b). It is noted that the FM response is not degraded by using stripe geometry structure.

The enhancement of relaxation oscillation frequency of stripe geometry semiconductor lasers can be understood from the modified expression of relaxation oscillation frequency [6], $\omega_{\mathrm{f}}^{2}$,

$$
\omega_{\mathrm{f}}^{2}=\frac{\left\langle\mathrm{P}_{\mathrm{o}}\right\rangle}{\tau_{\mathrm{p}}}\left(\Gamma_{\mathrm{o}} \frac{\partial \mathrm{G}_{\text {eff }}}{\partial \mathrm{N}}+\mathrm{G}_{\mathrm{o}} \frac{\partial \Gamma_{\text {eff }}}{\partial \mathrm{N}}\right)
$$

where $\left\langle\mathrm{P}_{0}\right\rangle, \mathrm{G}_{0}$ and $\Gamma_{\mathrm{o}}$ are the average photon density, material gain and confinement factor at steady state, respectively. $\partial \mathrm{G}_{\text {eff }} / \partial \mathrm{N}$ and $\partial \mathrm{r}_{\text {eff }} / \partial \mathrm{N}$ are the effective differential gain and differential confinement factor. $\tau_{p}$ is the effective photon lifetime.

In strong index guided devices, the carrier induced refractive index change in the active region has almost negligible effect on the optical field profile and $\partial \Gamma_{\text {eff }} / \partial \mathrm{N}$ is approximately equal to zero. However, in gain guided structure, the confinement factor is increased with the carrier concentration and therefore $\partial \Gamma_{\text {eff }} / \partial \mathrm{N}$ is positive. Hence, the time dependent lateral optical field has no effect on buried heterostructure lasers and a positive effect (increase $\omega_{f}$ ) on stripe geometry lasers.

The relaxation oscillation frequency of DFB lasers is also affected by the cavity loss $\left(=1 / \tau_{p}\right)$ and the total photon energy, $\left\langle\mathrm{P}_{\mathrm{O}}\right\rangle$, stored inside the active region [3]. These parameters are influenced by spatial hole burning. It is noted that $\lambda / 4$ devices with high $\kappa \mathrm{L}(>2.0)$ have relatively large relaxation oscillation frequency [3] because the total energy stored in large $\kappa \mathrm{L}$ device is relatively high and can compensate the variation of cavity loss $\left(1 / \tau_{p}\right.$ decreases as $\kappa L$ increases for $\lambda / 4$ devices). In gain guided devices, this situation will be more apparent because the existence of $\partial \Gamma_{\text {eff }} / \partial \mathrm{N}$ in equation(1) enhances the spatial hole burning effect.

The magnitude of $\partial \Gamma_{\text {eff }} / \partial \mathrm{N}$ is a function of stripe width and the optimized AM bandwidth can be obtained with a suitable stripe width. The relation between $\partial \Gamma_{\text {eff }} / \partial \mathrm{N}$ and the stripe width is shown in figure 3. It is shown that the value of $\partial \Gamma_{\text {eff }} / \partial \mathrm{N}$ is increased with the reduction of stripe width. However, device with narrow stripe width is not desired because of high threshold current and selfsustained pulsation.

In conclusion, the results show that the small signal AM bandwidth of $\lambda / 4$ DFB lasers can be enhanced by using stripe geometry structure. This is because the time dependent lateral optical field profile (i.e. $\partial \Gamma_{\text {eff }} / \partial \mathrm{N}$ ) enhances the relaxation oscillation frequency of stripe geometry lasers. 
In addition, the modulation bandwidth can be further increased by using devices with large $\kappa\left(>50 \mathrm{~cm}^{-1}\right)$. The total AM bandwidth can be increased by more than $3 \mathrm{GHz}$.

\section{References}

[1] N. Chinone, K. Aiki, M. Nakamura and R. Ito, 'Effects of lateral mode and carrier density profile on dynamic behaviors of semiconductor lasers', IEEE J. Quantum Electron., vol QE-14, no.8, pp.625-631, 1978.

[2] R. Olshansky, P. Hill, V.A. Lanzisera and W. Powazinik, 'Frequency response of $1.3 \mu \mathrm{m}$ InGaAsP high speed semiconductor lasers', IEEE J. Quantum Electron., vol. QE-23, p1410$1418,1987$.

[3] S.F. Yu, R.G.S. Plumb, L.M. Zhang, M.C. Nowell \& J.E. Carroll, 'Large signal dynamic behavior of distributed feedback lasers including lateral effects', IEEE J. Quantum Electron., Vol. QE-30, no.8, pp.1740-1750, 1994.

[4] S.F. Yu, 'Static and dynamic response of narrow-stripe distributed feedback semiconductor lasers', submitted for publication.

[5] L.M. Zhang and J.E. Carroll, 'Enhanced AM and FM modulation response of complex coupled DFB lasers', IEEE Photo. Technol. Lett., vol.5, pp.506-508, 1993.

[6] J.T. Verdeyen,'Laser electronics', Prentic-Hall International Editions, 2nd edition, chapter 11, 1989. 


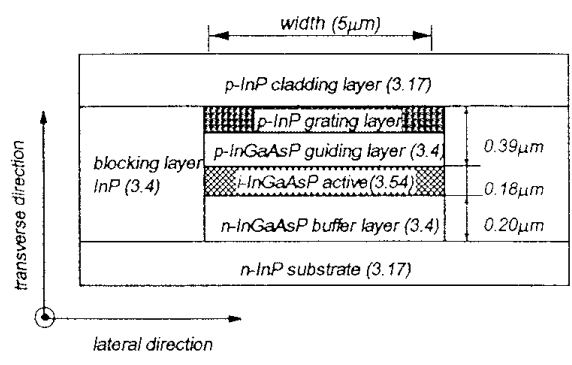

(a)

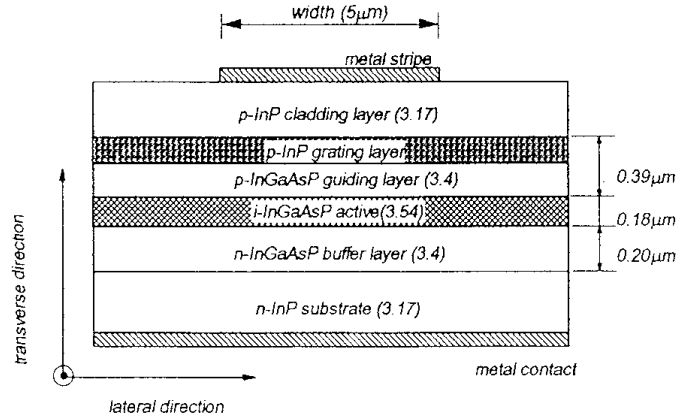

(b) Stripe Geometry

Figure 1 The cross section of the DFB lasers with (a) buried heterostructure and (b) stripe geometry.
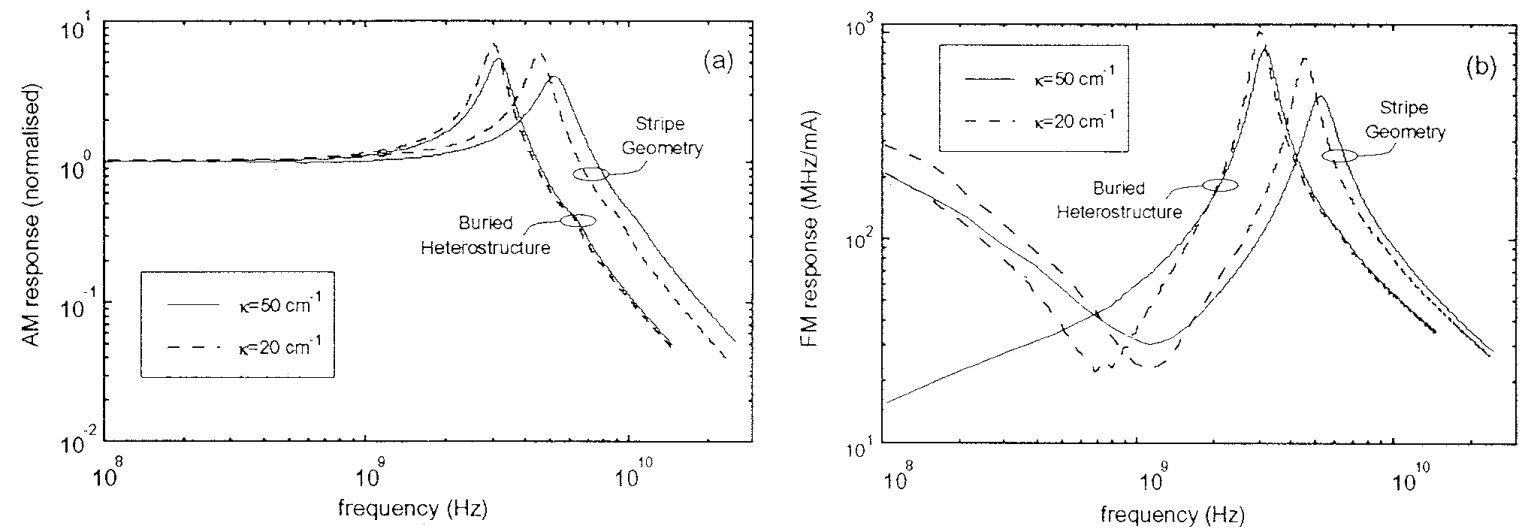

Figure 2 Small signal dynamic responses of buried heterostructure and stripe geometry $\lambda / 4$ DFB lasers. (a) Normalised AM response and (b) FM response.

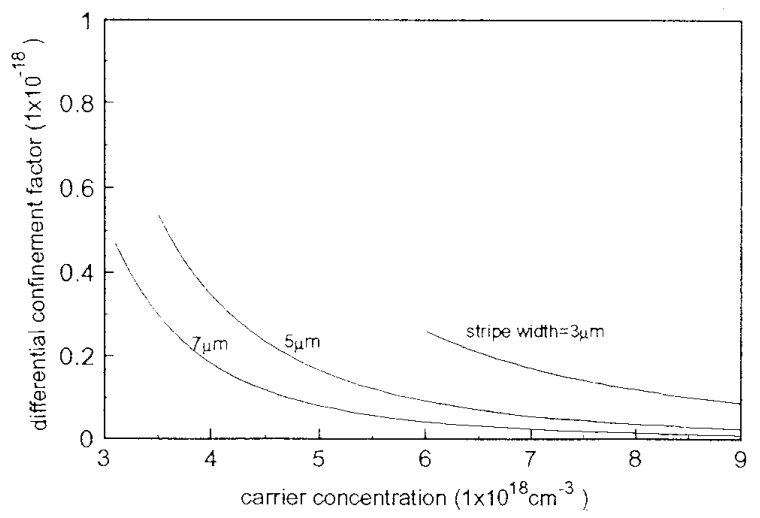

Figure 3 Calculated differential optical confinement factor as a function of carrier concentration and stripe width. 